Noname manuscript No.

(will be inserted by the editor)

\title{
Space-based infrared interferometry to study exoplanetary atmospheres
}

\author{
D. Defrère ${ }^{1}$, A. Léger ${ }^{2}$, O. Absil ${ }^{1}$, \\ C. Beichman ${ }^{3}$, B. Biller ${ }^{4}$, W.C. Danchi ${ }^{5}$, \\ K. Ergenzinger ${ }^{6}$, C. Eiroa $^{7}$, S. Ertel ${ }^{8}$, \\ M. Fridlund ${ }^{9,16}$, A. García Muñoz ${ }^{10}$, \\ M. Gillon ${ }^{1}$, A. Glasse ${ }^{11}$, M. Godolt ${ }^{10}$, \\ J.L. Grenfell ${ }^{12}$, S. Kraus ${ }^{13}$, L. Labadie ${ }^{14}$, \\ S. Lacour ${ }^{15}$, R. Liseau ${ }^{16}$, G. Martin ${ }^{17}$, \\ B. Mennesson ${ }^{18}$, G. Micela ${ }^{19}$, S. Minardi ${ }^{20}$, \\ S.P. Quanz ${ }^{21}$, H. Rauer ${ }^{10,12}$, S. Rinehart ${ }^{5}$, \\ N.C. Santos ${ }^{22,23}$, F. Selsis ${ }^{24}$, J. Surdej ${ }^{1}$, \\ F. Tian $^{25}$, E. Villaver ${ }^{7}$, P.J. Wheatley ${ }^{26}$, \\ M. Wyatt ${ }^{27}$.
}

Received: date / Accepted: date

D. Defrère

Tel.: +32-4-3669758

E-mail: ddefrere@uliege.be

1 Space sciences, Technologies \& Astrophysics Research (STAR) Institute, University of

Liège, Liège, Belgium

. ${ }^{2}$ Institut d'Astrophysique Spatiale, Université de Paris-Sud, Orsay, France

. 3 NASA Exoplanet Science Institute, California Institute of Technology, Pasadena, California, USA

${ }^{4}$ Institute for Astronomy, University of Edinburgh, Edinburgh, United Kingdom

${ }^{5}$ NASA Goddard Space Flight Center, Exoplanets \& Stellar Astrophysics Laboratory, Greenbelt, USA

${ }^{6}$ Airbus Defence and Space GmbH

. 7 Dpto. Fisica Teorica, Universidad Autonoma de Madrid, Canto-blanco, 28049 Madrid, Spain

. 8 Steward Observatory, Department of Astronomy, University of Arizona, Tucson, Arizona, USA

. ${ }^{9}$ Leiden Observatory, University of Leiden, PO Box 9513, NL-2300 RA, Leiden, the Netherlands

. ${ }^{10}$ Technische Universität Berlin, Berlin, Germany

. 11 Astronomy Technology Centre, United Kingdom

. ${ }^{12}$ Institute for Planetary Research, German Aerospace Center, Berlin, Germany

. 13 School of Physics and Astronomy, University of Exeter, Exeter, United Kingdom

14 University of Cologne, Germany

. ${ }^{15}$ LESIA, Observatoire de Paris, PSL Research University, 92195 Meudon Cedex, France

. ${ }^{16}$ Department of Space, Earth and Environment, Chalmers University of Technology, On-

sala Space Observatory, 43992 Onsala, Sweden

. 17 Institut de planétologie et d'astrophysique de Grenoble, Grenoble, France

. 18 Jet Propulsion Laboratory, USA 
Abstract The quest for other habitable worlds and the search for life among them are major goals of modern astronomy. One way to make progress towards these goals is to obtain high-quality spectra of a large number of exoplanets over a broad range of wavelengths. While concepts currently investigated in the United States are focused on visible/NIR wavelengths, where the planets are probed in reflected light, a compelling alternative to characterize planetary atmospheres is the mid-infrared waveband $(5-20 \mu \mathrm{m})$. Indeed, mid-infrared observations provide key information on the presence of an atmosphere, the surface conditions (e.g., temperature, pressure, habitability), and the atmospheric composition in important species such as $\mathrm{H}_{2} \mathrm{O}, \mathrm{CO}_{2}, \mathrm{O}_{3}, \mathrm{CH}_{4}$, and $\mathrm{N}_{2} \mathrm{O}$. This information is essential to investigate the potential habitability of exoplanets and to make progress towards the search for life in the Universe. Obtaining high-quality mid-infrared spectra of exoplanets from the ground is however extremely challenging due to the overwhelming brightness and turbulence of the Earth's atmosphere. In this paper, we present a concept of space-based midinfrared interferometer that can tackle this observing challenge and discuss the main technological developments required to launch such a sophisticated instrument.

Keywords Space Interferometer · Infrared astronomy · Darwin · TPF-I . Exoplanet $\cdot$ Habitability $\cdot$ Bio-signatures

\section{Introduction}

The goal of finding habitable planets and even planets with signs of (primitive) life around other stars is extremely challenging and requires a variety of complementary observations. Experience gained so far in exoplanet atmospheric research shows that a broad wavelength coverage and sufficiently high spectral resolution are required to break the degeneracies in composition and climate associated with retrieval of atmospheric spectra. In that regard, an important wavelength regime is the mid-infrared $(5-20 \mu \mathrm{m})$. It provides data to measure key planetary parameters, such as the size, temperature, the presence of an atmosphere, as well as the presence of important atmospheric molecules such as $\mathrm{H}_{2} \mathrm{O}, \mathrm{CO}_{2}, \mathrm{O}_{3}, \mathrm{CH}_{4}$, and $\mathrm{N}_{2} \mathrm{O}$ (see Table 1). From an observational

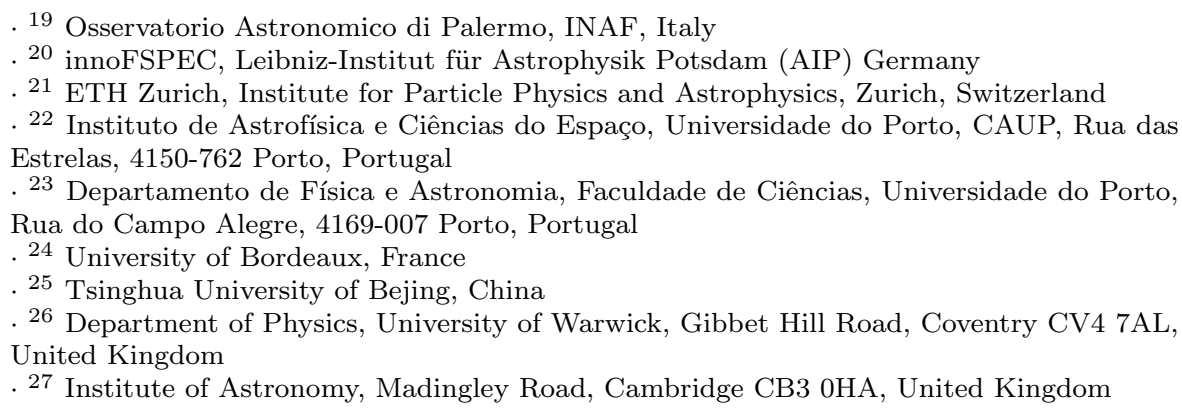


Table 1 Information on planets that can be obtained from low-resolution ( $\mathrm{R} \simeq 20)$ midinfrared observations $(5-20 \mu \mathrm{m})$. Two continuum (Cont.) bands (2 and 3) are also given, where in a cloud-free atmosphere, emission from the surface might be seen (data from Des Marais et al 2002 Seager et al 2016, Airapetian et al 2017). Values given for $\mathrm{N}_{2} \mathrm{O}$ and NO are approximative. The wavelength columns $\left(\lambda_{\mathrm{MIN}}, \lambda_{\mathrm{MAX}}\right.$, and $\left.\lambda_{\mathrm{AVG}}\right)$ are given in microns. $\mathbf{R}$ gives the corresponding spectral resolution.

\begin{tabular}{|c|c|c|c|c|c|c|}
\hline & Information on planet & Species & $\lambda_{\mathrm{MIN}}$ & $\lambda_{\text {MAX }}$ & $\lambda_{\mathrm{AVG}}$ & $\mathrm{R}$ \\
\hline 1 & Orbit characteristics & Cont. 1 & 6.00 & 20.0 & 13.0 & 1 \\
\hline \multirow[t]{2}{*}{2} & \multirow{2}{*}{$\begin{array}{l}\text { Combination of temperature, } \\
\text { radius, and albedo }\end{array}$} & Cont. 2 & 10.1 & 12.4 & 11.2 & 5 \\
\hline & & Cont. 3 & 8.16 & 9.24 & 8.67 & 8 \\
\hline \multirow[t]{5}{*}{3} & \multirow[t]{5}{*}{ Existence of atmosphere } & Cont. 1 & 6.00 & 20.0 & 13.0 & 1 \\
\hline & & $\mathrm{CO}_{2}$ & 9.07 & 9.56 & 9.31 & 19 \\
\hline & & & 10.1 & 10.7 & 10.4 & 16 \\
\hline & & & 13.3 & 17.0 & 15.0 & 4 \\
\hline & & $\mathrm{NO}$ & 5.1 & 5.5 & 5.3 & 20 \\
\hline \multirow[t]{2}{*}{4} & \multirow[t]{2}{*}{ Presence of water } & $\mathrm{H}_{2} \mathrm{O}$ & 6.67 & 7.37 & 7.00 & 10 \\
\hline & & & 17.4 & 25.0 & 20.5 & 3 \\
\hline \multirow[t]{4}{*}{5} & \multirow[t]{4}{*}{ Suggestion of life } & $\mathrm{CH}_{4}$ & 7.37 & 7.96 & 7.65 & 13 \\
\hline & & & 7.37 & 8.70 & 7.98 & 6 \\
\hline & & $\mathrm{N}_{2} \mathrm{O}$ & 7.50 & 9.00 & 7.25 & 10 \\
\hline & & $\mathrm{O}_{3}$ & 9.37 & 9.95 & 9.65 & 17 \\
\hline
\end{tabular}

standpoint, it also has the considerable advantage of offering a more favorable planet/star contrast than at visible wavelengths $\left(10^{-7}\right.$ vs $\left.10^{-10}\right)$ for an EarthSun system.

Mid-infrared observations from the ground are however very challenging due to the turbulence and brightness of the atmosphere. For instance, the atmosphere is approximately 10 billion times brighter than a $300 \mathrm{~K}$ Earth-sized planet located at $10 \mathrm{pc}$ when observed with an 8-m telescope. Overcoming the background photon noise limit alone requires prohibitively long integration times to study rocky exoplanets, even around nearby stars. In addition, because the Earth's atmosphere is mostly opaque at the wavelengths corresponding to major molecular absorption features (such as $\mathrm{H}_{2} \mathrm{O}$ and $\mathrm{CO}_{2}$ ), searching for the broad spectral signatures of major molecular species in planetary atmospheres will generally be very difficult from the ground. To overcome these issues, access to space is mandatory. In the short term, the study of transiting systems is highly promising for planets larger than a few Earth radii and will be a major focus for the James Webb Space Telescope (Beichman et al 2014). Nevertheless, the rarity of nearby planetary systems suitably aligned to produce a transit, the short duration of the transit, and the noise produced by the host star means that the study of the atmospheres of terrestrial planets, especially those orbiting Sun-like stars, is probably unachievable via this technique. Thus, techniques which separate the light of the planet from the glare of the host star and can characterize exoplanets with any orbital configuration are essential. 
In order to spatially resolve the systems closest to Earth (within $10 \mathrm{pc}$ ) in the mid-infrared, an aperture of at least $80 \mathrm{~m}$ in diameter would however be required and this is presently not feasible. A way to increase the spatial resolution is to use an interferometer as initially proposed by Bracewell (1978) and significantly improved by Angel et al (1986). The concept of such an instrument was extensively studied in the 1990s and 2000s by both ESA and NASA. In Europe, ESA focused mainly on the DARWIN project (e.g., Léger et al 1996, Cockell et al 2009), which consisted of a space-based flotilla of midinfrared telescopes using nulling interferometry as the measurement principle. In the United states, a similar concept, called the Terrestrial Planet Finder Interferometer (TPF-I, Angel et al 1997), was considered as the final piece of NASA's ambitious Navigator program to characterize Earth-like exoplanets (Lawson and Traub 2006). Between 1996 and 2007, considerable efforts have been carried out by both agencies to define the best mission design and to advance the technologies required for such an ambitious endeavor. Several mission architectures were proposed, key enabling technologies were developed and demonstrated on laboratory test-benches, and advanced data reduction techniques were investigated. These efforts, which resulted in hundreds of articles in the technical literature, culminated in 2007 with the convergence and consensus on a mission architecture called the Emma X-array (Cockell et al 2009). In parallel, both agencies also appointed teams to investigate the scientific issues related to the search for life on exoplanets (e.g., Lawson et al 2007; Fridlund et al 2010). Some of the key questions are: What are the atmospheric compositions of rocky exoplanets? Are they habitable? What is a biosignature? Do exoplanets show signs of biological activity? How common is a planet like Earth? How do rocky planets form? While most DARWIN and TPF-I activities stopped after 2007 because of fundings reasons, these scientific questions are still a central focus. Today, the exoplanet landscape has greatly changed compared to 2007 but the general consensus in the exoplanet community is still the same: mid-infrared spectra will be required to tackle these fundamental questions (see e.g., Fujii et al 2018).

\section{Studying planetary atmospheres in the mid-infrared}

\subsection{Presence of an atmosphere and basic planetary properties}

For any atmospheric composition, monitoring the variations of thermal emission of an exoplanet during its orbital motion provides a fundamental constraint on meridional transport (hence atmospheric mass) as well as on cloud coverage (e.g., Selsis 2004). With an increasing number of thermal emission bands monitored, additional planetary properties can be inferred or constrained by orbital photometry: rotation, albedo, obliquity, radius (Selsis et al 2011: Cowan et al 2012, Maurin et al 2012, Selsis et al 2013), presence of a large satellite (Moskovitz et al 2009), response to eccentricity (Bolmont et al 2016). 
Thermal phase curves have successfully been used to characterize unresolved transiting (Knutson et al 2007; Stevenson et al 2014) and even non-transiting giant planets (Crossfield et al 2010) but the stellar variability and the required photometric precision make these measurements very difficult. With directly imaged planets, this method will show its full potential. Although the inner working angle will limit the access to the smallest phase angles, only a moderate photometric precision of $\sim 10 \%$ will be required to achieve a crucial diagnostic and first classification of the targets. Another strength of the method comes from the favorable distribution of orbit orientations. For the median inclination of randomly oriented orbits $\left(60^{\circ}\right)$, the amplitude of phase curves is only decreased by $10 \%$ compared with the maximum variations, reached for a $90^{\circ}$ inclination (Maurin et al 2012). Only a few observations covering one orbit would be sufficient to start deriving constraints on the climate and several low-resolution or broadband observations would be necessary to resolve the orbital motion of the planet. Note finally that a by-product of thermal emission monitoring is the orbit characteristics, such as semi-major axis, period, inclination, and position angle.

\subsection{Surface conditions, atmospheric composition, and habitability}

In addition to the constraints from orbital broadband photometry, mid-infrared spectroscopy is fundamental to investigate the nature of planetary atmospheres by detecting spectral features, constraining the temperature and pressure structure, the cloudiness and by determining whether the planet could potentially be habitable. Indeed, several important species have mid-infrared spectral signatures that can be detected at low to medium spectral resolving power (e.g., $\mathrm{H}_{2} \mathrm{O}, \mathrm{CO}_{2}, \mathrm{O}_{3}, \mathrm{CH}_{4}, \mathrm{~N}_{2} \mathrm{O}$ ). The wavelength (minimum, maximum, and average) and corresponding spectral resolution for each spectral feature are given in Table 1). In addition, surface conditions (temperature and pressure) can be characterised relatively well from mid-infrared observations (to within $\sim 10 \mathrm{~K}$ at $3-\sigma$ ) with $\mathrm{S} / \mathrm{Ns}$ between 10 and 30, depending on spectral resolution (von Paris et al 2013). By observing a large number of Habitable Zone (HZ) rocky exoplanets, it will be possible to correlate the concept of habitability with key parameters and processes like spectral type of the parent star, degree of stellar activity, the temperature/pressure structure of the atmosphere, gaseous composition, the circulation and heat transfer of the atmosphere, the atmospheric chemistry and photochemistry, the outgassing of atmospheric species, and the presence of a magnetic field.

\subsection{Understanding the concept of biosignature}

A biosignature can be defined as "an observable feature of a planet, such as its atmospheric composition, that our present models cannot reproduce when including the abiotic physical and chemical processes we know about" (Léger 
et al 2011). In a recent review, Catling et al (2018) define a biosignature as "any phenomenon, substance, or group of substances that provides evidence of the presence of life." These include biosignature gases in the atmosphere, oxygen $\left(\mathrm{O}_{2}\right)$, ozone $\left(\mathrm{O}_{3}\right)$, methane $\left(\mathrm{CH}_{4}\right)$, nitrous oxide $\left(\mathrm{N}_{2} \mathrm{O}\right)$, and methyl chloride $\left(\mathrm{CH}_{3} \mathrm{Cl}\right)$; and a surface biosignature, the vegetation "red edge," a unique reflectance spectrum from plant leaves and a sign of oxygenic photosynthesis. Over the past few years, researchers have probed the efficacy of these signs of biogenic gases and photosynthesis for exoplanets and identified additional candidates. In particular, Schwieterman et al (2018) have presented an exhaustive review of the mechanisms, sources, sinks, and environmental by-products for gas and surface biosignature candidates to date.

While the scientific community keeps improving the list of potential biosignature species (e.g., Seager et al 2016; Kiang et al 2018), it appears clear that our theoretical models are currently limited and the emerging vision is that studying the atmospheres of a large number of exoplanets will be required to provide an essential context for interpreting possible detections of bio-signatures. A good approach could be to create the planetary equivalent of a "Hertzsprung-Russel" diagram including species suggestive of life. Only after observing a sufficient number of planetary atmospheres, will it be possible to identify possible anomalies in this diagram that cannot be explained without the presence of life. To make progress in that direction, the mid-infrared regime has a key role to play since it contains spectral signatures of important molecules such as $\mathrm{H}_{2} \mathrm{O}, \mathrm{CO}_{2}, \mathrm{O}_{3}, \mathrm{~N}_{2} \mathrm{O}$, and $\mathrm{CH}_{4}$. In fact, the triple signature (i.e., $\mathrm{O}_{3}, \mathrm{CO}_{2}, \mathrm{H}_{2} \mathrm{O}$ ) was considered as the most robust indicator for life at the time of the DARWIN/TPF studies and would still today be considered as a serious hint of biological activity. The advantage of $\mathrm{O}_{3}$ over $\mathrm{O}_{2}$ is that $\mathrm{O}_{3}$ is a highly sensitive indicator for the existence of even a trace amount of $\mathrm{O}_{2}$ and hence easier to detect at low $\mathrm{O}_{2}$ concentrations (Des Marais et al 2002). It is also difficult to produce abiotic $\mathrm{O}_{3}$ if water is present, due to catalytic cycles initiated by water photolysis, which removes $\mathrm{O}_{3}$. An in-depth review of $\mathrm{O}_{2}$ as a biosignature, rigorously examining the nuances of false positives and false negatives for evidence of life can be found in Meadows et al (2018).

\section{Space-based interferometer concept}

\subsection{Extracting the planetary photons by nulling interferometry}

The basic principle of nulling interferometry is to combine the beams coming from two telescopes with a 180 degree phase shift so that a dark fringe appears on the line of sight towards the hosts star, which strongly suppresses its light. On the other hand, off-axis emission, such as that of a planet, can be transmitted by optimizing the baseline length since the nearest bright fringe 
is located $\lambda / 2 /$ baseline from the dark fringe. However, even when the stellar emission is sufficiently reduced, it is generally not possible to detect Earth-like planets with a static array configuration, because their emission is dominated by the thermal contribution of a series of other extraneous and generally dominant signals originating from the telescope itself (thermal background, readout noise), material in the Solar system (the local zodiacal emission), or around the target star (exozodiacal light). This is the reason why Bracewell proposed to rotate the interferometer so that the planetary signal gets modulated by alternatively crossing high and low transmission regions, while the stellar signal and the background emission remain constant. The planetary signal can then be retrieved by synchronous demodulation. This modulation technique is in many ways similar to the use of a chopper wheel that allows the detection of infrared sources against a thermal background and/or drifting detector offsets.

\subsection{The Emma X-array configuration}

During the DARWIN/TPF-I studies, it was quickly realized that the interferometric array cannot be rotated sufficiently fast to mitigate low frequency instrumental drifts to a level sufficiently low to enable the observation of an Earth-like exoplanet around a Sun-like star (e.g., Lay 2004). A solution proposed to overcome this problem is to use more than two telescopes and phase chopping, which consists in synthesizing two different transmission maps with the same telescope array, by applying different phase shifts in the beam combination process. In addition to allowing more precise differential measurements, it is also possible to isolate the planetary signal from the contributions of symmetric brightness emissions such as the star, local zodiacal cloud, exozodiacal cloud, stray light, thermal, or detector gain. After the investigation of several interferometer architectures (e.g., Angel and Woolf 1997; Mennesson and Mariotti 1997), two array architectures have been thoroughly investigated by ESA during two parallel assessment studies carried out by EADS Astrium and Alcatel-Alenia Space in 2005-2006: the four-telescope X-array and the ThreeTelescope Nuller (TTN, Karlsson et al 2004). These studies included the launch requirements, payload spacecraft, and the ground segment during which the actual mission science would be executed. Almost simultaneously, NASA/JPL initiated a similar study for TPF-I and focused in particular on the Dualchopped Bracewell (Lay 2004) and X-array configurations. These efforts on both sides of the Atlantic have finally resulted in a convergence and consensus on mission architecture, the so-called non-coplanar (aka Emma-type) X-array. The baseline design consisted in four collector spacecraft, flying in rectangular formation and feeding light to the beam combiner spacecraft located approximately $1200 \mathrm{~m}$ from the array. This arrangement makes available baselines up to $170 \mathrm{~m}$ for nulling measurements and up to $500 \mathrm{~m}$ for the general astrophysics program (constructive imaging with an angular resolution of 4 mas at $10 \mu \mathrm{m}$ ). 


\subsection{Exoplanet yield}

The number of planetary atmospheres that can be studied with such an instrument is a critical metric to estimate the science return of the mission. During the DARWIN/TPF studies, the exoplanet yield has been extensively studied and cross-validated between ESA and NASA using various assumptions on the existing exoplanet population and prevalence of exozodiacal dust, which were both unknown at that time (e.g., Defrère et al 2010). Today, the occurrence of HZ rocky exoplanets has been measured by Kepler (e.g., Winn and Fabrycky 2015) and the prevalence of exozodiacal dust has been very well constrained by ground-based nulling interferometers (Mennesson et al 2014, Ertel et al. in prep). It is therefore possible to predict the exoplanet yield of direct imaging instruments more precisely. Recently, based on planet occurrence statistics from Kepler and using Monte-Carlo simulations, Kammerer and Quanz (2017); Quanz et al (2018) estimated that $\sim 315_{-77}^{+113}$ exoplanets with radii between $0.5 \mathrm{R}_{\oplus}$ and $6 \mathrm{R}_{\oplus}$ can be detected during $\sim 0.52$ years of mission time assuming four 2-m apertures and throughputs 3.5 times worse than those for the JWST and $40 \%$ overheads. Approximately 85 planets could be habitable (radii between $0.5 \mathrm{R}_{\oplus}$ and $1.75 \mathrm{R}_{\oplus}$ and equilibrium temperatures between $200 \mathrm{~K}$ and $450 \mathrm{~K}$ ) and would be prime targets for follow-up spectroscopic observations. While this exoplanet yield can be interpreted as an upper limit because no exozodiacal dust was assumed in this study, new results from the NASA's Hunt for Observable Signatures of Terrestrial planetary Systems (HOSTS, Danchi et al 2014) survey on the Large Binocular Telescope Interferometer (LBTI, Hinz et al 2016) suggest that exozodiacal dust disks would not be a major source of noise (Ertel et al 2018a b). New science yield estimates based on HOSTS upper limits are currently under study.

\subsection{Technology state-of-the-art}

\subsubsection{Formation flying}

Formation flying is a key technology for the deployment and success of a spacebased interferometer. Remarkable advances in technology have been made in Europe in recent years with the space-based demonstration of this technology by the PRISMA mission (D'Amico et al 2012). PRISMA demonstrated a sub-cm positioning accuracy between two spacecraft (see Figure 1), mainly limited by the metrology system (GPS and RF). The launch of ESA's PROBA3 mission in 2020 will provide further valuable free-flyer positioning accuracy results (sub-mm), which exceeds the requirements for a space-based interferometer that relies on fast pathlength correctors for precise Optical Path Delay (OPD) control. Extending the flight-tested building-block functionality from a distributed two-spacecraft instrument to an instrument with more spacecraft mainly relies on the replication of the coordination functionality and does not present additional complexity in terms of procedures according to 


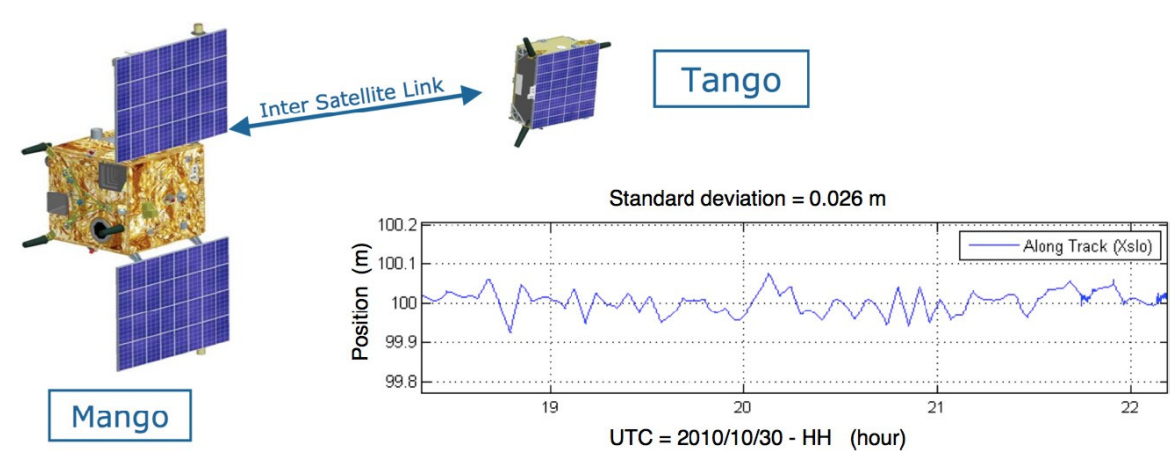

Fig. 1 Demonstration of Formation Flying between the two PRISMA spacecraft, Tango and Mango. A distance of $100 \mathrm{~m}$ was maintained during four hours with a standard deviation of few centimeters (see bottom right panel), limited by the accuracy of the radio frequency sensors. ESA's Proba 3 instrument, scheduled for a launch in 2021, should reduce this error to a few 100 microns. (C) Swedish Space Corporation, CNES and DLR.

the PRISMA navigation team. While formation flying can then be considered to have reached a technical readiness level (TRL) of 9 once PROBA-3 has flown, an uncertainty remains regarding fuel usage and the possible lifetime of such a mission.

\subsubsection{Spatial filters}

Spatial filters are optical devices which significantly reduce the optical aberrations in wavefronts. Consequently, they are very important for nulling interferometry, making extremely deep nulls possible. To provide spatial filtering over large bandwidths, a variety of techniques can be applied, including singlemode fiber optics, photonic crystal fibers, or integrated optics. Developments of single-mode fibers for the mid-infrared were funded by NASA between 2003 and 2008 (Ksendzov et al 2007, 2008). Fiber optics made of chalcogenide and silver halide materials have been demonstrated to yield $25 \mathrm{~dB}$ or more rejection of higher-order spatial modes at $10 \mu \mathrm{m}$, but they would require the division of the $6-20 \mu \mathrm{m}$ band into two parts. Although this performance is sufficient for flight, it would be greatly advantageous to improve the throughput of these devices and to test them over the full wavelength range in cryogenic conditions. Spatial filter technology would then be at TRL 5. The spatial filtering capabilities of photonic crystal fibers should also be investigated for use at mid-infrared wavelengths, because of the improved throughput that they may provide and the possibility to cover the whole wavelength range with a single device. Note finally that, given the recent developments in wavefront control with extreme adaptive optics systems (e.g., Jovanovic et al 2015), it is not clear whether such a filtering technology will be required. This should be addressed in the future. 


\subsubsection{Beam combination}

Classical optical designs of four-telescope nulling beam combiners (Martin and Booth 2010) have been demonstrated to flight requirement levels albeit at room temperature and using signals much stronger than those from astronomical sources. Recently, a very promising grating nuller approach was proposed and shown to achieve nulls of $4 \times 10^{-5}$ over the full $18 \%$ bandwidth K-band (Martin et al 2017). Alternatively, integrated optics (IO) beam combination can be achieved by a network of single-mode waveguides embedded in a cm-scale glass chip. This solution bypasses complex optical interferometric trains sensitive to vibrational, thermal and mechanical stress, hence reducing the risk associated with bulky science instruments. As for fibers, IO can furthermore achieve wavefront cleaning to mitigate phase errors (Wallner et al 2002, Mennesson et al 2002). Silica-based IO solutions are now being successfully implemented in operating near-infrared 4-telescope interferometers (Eisenhauer et al 2011 Le Bouquin et al 2011) and have also been investigated for nulling applications in the near-infrared at $1.5 \mu \mathrm{m}$ (Weber et al 2004, Errmann et al 2015) with stable nulls down to $10^{-4}$ over $5 \%$ bandwidth. Since silica glasses are opaque to IR radiation for $\lambda>2 \mu \mathrm{m}$, the extension of the IO approach to the midinfrared in the 3-30 $\mu \mathrm{m}$ range requires an adequate material and technological platform to manufacture high quality optical chips.

\subsubsection{Starlight suppression}

A considerable expertise has been developed in the field of starlight suppression over the past 20 years, both in academic and industrial centers across the globe. These efforts culminated with laboratory demonstrations of the recombination scheme to flight requirements at the Jet Propulsion Laboratory (JPL) in the US. In particular, mid-infrared nulls of $10^{-5}$ were achieved with both the Adaptive Nuller (Peters et al 2010) and the planet detection testbed (Martin et al 2012, see Figure 2), but with fluxes much higher than those expected from stars and planets allowing working at room temperature without being disturbed by the thermal emission of the environment. In parallel, the operation of high-precision ground-based interferometers has matured in both Europe and the US. In particular, Europe has gained a strong expertise in the field of fringe sensing, tracking, and stabilization with the operation of the Very Large Telescope Interferometer (VLTI). In the United states, considerable technical expertise was gained by operating several nulling interferometers such as the Keck Interferometer Nuller (KIN, Colavita et al|2009), the Palomar Fiber Nuller (PFN, Mennesson et al 2011b), and the Large Binocular Telescope Interferometer (Hinz et al 2016). All have produced excellent scientific results (e.g., Mennesson et al 2014 : Defrère et al 2015), which have pushed high-resolution mid-infrared imaging to new limits (Defrère et al 2016). Innovative data reduction techniques have also been developed to improve the 


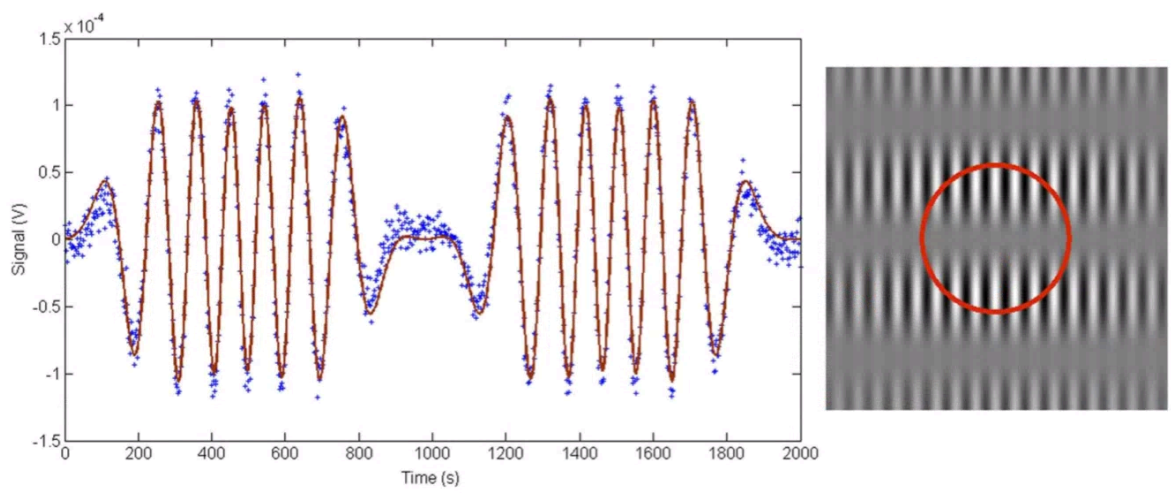

Fig. 2 The left panel shows the planet signal detected with the Planet Detection Testbed (Martin et al 2012). Each point is an item of data from the 2-s chop cycle and the whole trace shows the null signal obtained over a 360 degree effective rotation of the interferometer array. The line is a fit to the signal from a planet at a nominal angular radius of $6.35 \times 10^{-7} \mathrm{rad}$ (or 132 mas) from the star. By comparison, the equivalent angular fringe distance from the short baseline is $4.7 \times 10^{-7} \mathrm{rad}$. Near the center and at the ends of the plot, the planet crosses the null fringe. The right panel shows the equivalent sensitivity map of the interferometer array. Array rotation causes the planet location to orbit (solid line) around the central null fringe (gray), and thus its signal is modulated both by the higher frequency fringes on the long baseline and by the chopping. Note that this demonstration was realized at room temperature with high flux levels. Technological developments are required to repeat this demonstration in cryogenic conditions with optical fluxes similar to those expected from astronomical sources (see Section 4.3).

accuracy of nulling instruments (Hanot et al 2011, Mennesson et al 2011a) but more work is required to adapt this technique to four-telescope configurations.

\section{Prospects}

\subsection{Current context}

Because it is a very challenging observational task partially requiring further technological developments, the spectroscopic characterisation of small $\mathrm{HZ}$ exoplanets by direct imaging is currently not possible with existing instruments. Future instruments considered to achieve this goal cover generally two distinct and complementary wavelength ranges: the visible, which favors the angular resolution, and the mid-infrared, which favors the contrast. Focusing first on the mid-infrared regime, we can see in Figure 3 that none of current or foreseen instruments can approach a space-based nulling interferometer when it comes to achieving the necessary sensitivity at a small angular separation from the parent star. In the case of JWST, the impressive sensitivity provided by the large collecting area $\left(25 \mathrm{~m}^{2}\right)$ and cold $(40 \mathrm{~K})$ telescope optics can only be utilized by coronagraphs which are expected to achieve a best case contrast at $10.6 \mu \mathrm{m}$ of $10^{-4}$ to $10^{-5}$, for separations larger than 0.5 to 1.0 arcsecond (Boccaletti et al 2015). With such performances, the detection of warm and 


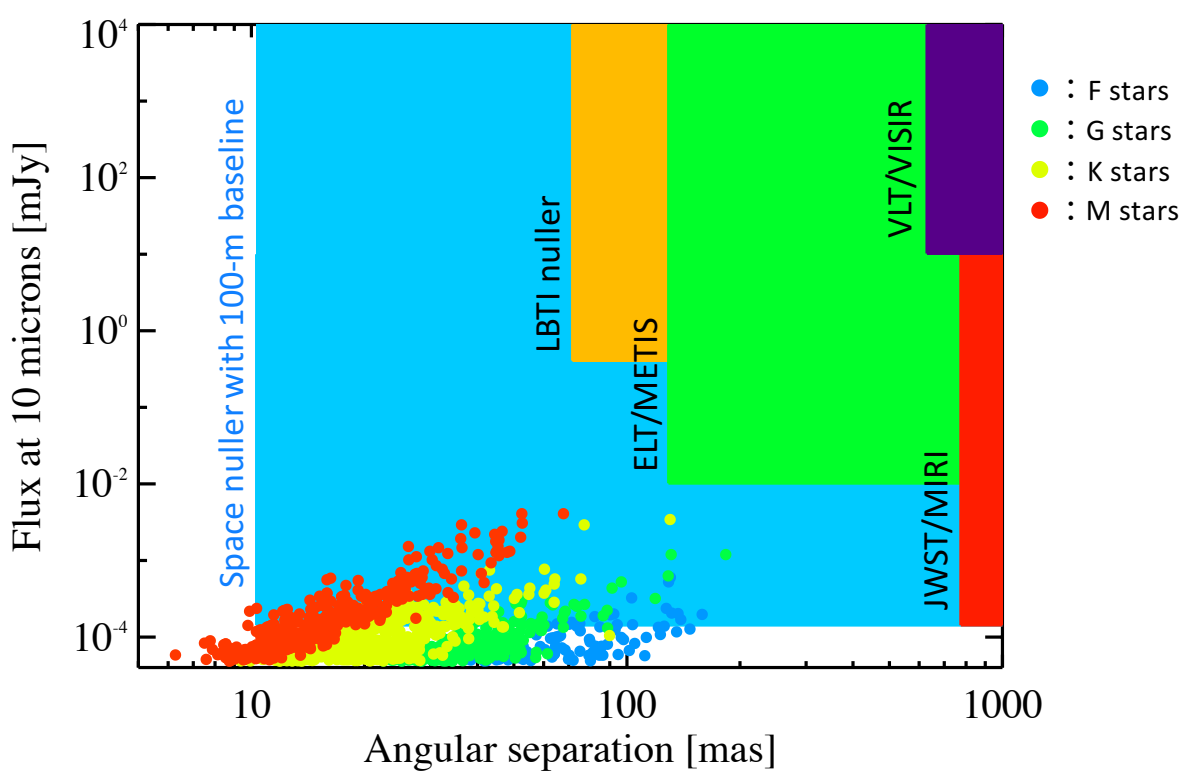

Fig. 3 Flux at $10 \mu \mathrm{m}$ as a function of angular separation of putative $300 \mathrm{~K}$ blackbody planets located in the middle of the $\mathrm{HZ}$ of nearby single main-sequence stars (DASSC catalog, Kaltenegger et al 2010). The 10- $\sigma$ sensitivity in one day of current and planned mid-infrared instruments is indicated by the colored rectangles (values scaled from Brandl et al 2014) and compared to that of a four 2m-aperture space-based nulling instrument (see blue rectangle, assuming no exozodiacal dust). Note that this plot does not take into account aperture masking modes, which improve the angular resolution but reduce the sensitivity.

young exo-Jupiters is the closest that the JWST/MIRI instrument can approach to this project's goal of exo-Earth characterisation. For the E-ELT, the massive gain in collecting area $\left(980 \mathrm{~m}^{2}\right)$ compared to the JWST offsets the impact of having warm optics to give comparable sensitivity limits for the METIS instrument, operating at 3 to $19 \mu \mathrm{m}$. METIS (e.g., Brandl et al 2016) will be equipped with coronagraphs which can in principle achieve contrasts of $\sim 10^{-7}$ at separation of $\sim 0.7$ arcsecond necessary to directly image a putative exo-Earth orbiting $\alpha$ Cen and $\sim 10$ small planets ( 1 to $4 \mathrm{R}_{\oplus}$ ) with equilibrium temperatures between 200 and $500 \mathrm{~K}$ around the nearest stars Quanz et al 2015). However, achieving this performance in practice at a ground-based observatory where image quality and stability are dependent on an advanced adaptive optics system, will be challenging. Further, due to the scarcity of available photons, the measurement would be restricted to a photometric detection, with little hope of spectroscopic follow-up.

Regarding the characterisation of $\mathrm{HZ}$ exoplanets in the visible, NASA is currently studying two concepts in preparation for the 2020 US decadal survey in Astronomy: (i) LUVOIR, a 10-16 m segmented, visible light telescope designed for an ambitious program of general astrophysics as well as detection of Earth-sized exoplanets and characterisation of dozens to hundreds of 
nearby stars; and (ii) HabEx, a 4-8 m monolithic telescope optimized for detection of Earth-sized exoplanets and characterisation of a smaller number of systems using either a highly optimized coronagraph or possibly a star shade. Both missions will likely include spectroscopy in the visible to near-infrared of Earth-sized planets in the HZ of nearby stars, searching for signs of habitability $\left(\mathrm{H}_{2} \mathrm{O}\right)$ and bio-signature gases $\left(\mathrm{O}_{2}, \mathrm{O}_{3}\right)$. A possible near-infrared extension (up to $\sim 2.5$ microns) of these high contrast spectroscopic capabilities would help further establish whether these gases were created by biotic processes or not, i.e., looking for species such as $\mathrm{CO}_{2}, \mathrm{CO}, \mathrm{O}_{3}, \mathrm{CH}_{4}$, and $\mathrm{N}_{2} \mathrm{O}$. Scientifically, there is no clear advantage of one wavelength range over the other as described in various reviews (see e.g., Des Marais et al 2002; Fujii et al 2018). Note that mid-infrared observations may also be used to estimate the planetary radius, which otherwise remains unobservable unless the planet transits.

\subsection{Precursor concepts}

The path towards space-based interferometry is regularly discussed and this often involves the need for precursor missions (e.g., Rinehart et al 2016). In addition to free-flying demonstrators already discussed in Section 3.4.1] concepts of small-scale space-based infrared nulling interferometers were seriously considered both in Europe and in the US: the Fourier-Kelvin Stellar Interferometer (FKSI, Danchi et al 2008) and Pegase (Ollivier et al 2009). Opportunities for testing technologies and for pushing new developments also exist with current ground-based facilities. For instance, in addition to ongoing research with current instruments as discussed in Section 3.4.4, there is currently a plan to build a nulling interferometer for the VLTI (i.e., the Hi-5 project, Defrère et al 2018). There is also a science-driven, international initiative to develop the roadmap for a future ground-based facility that will be optimised to image planet-forming disks on the spatial scale where the protoplanets are assembled, which is the Hill Sphere of the forming planets. This Planet Formation Imager (PFI, Monnier et al 2016) is designed to detect and characterise protoplanets during their first $\sim 100$ million years and trace how the planetary population changes due to migration processes, unveiling the processes that determine the final architecture of exoplanetary systems. With $\sim 20$ telescope elements and baselines of $\sim 3 \mathrm{~km}$, the PFI concept is optimised for imaging complex scenes at mid-infrared wavelengths $(3-12 \mu \mathrm{m})$ and at 0.1 milliarcsecond resolution. This clearly complements the capabilities of a space interferometer that would be optimised to achieve the sensitivity and contrast required to characterise the atmospheres of mature exoplanets.

\subsection{Required technological developments}

The main remaining technological challenge is the implementation of a cryogenic interferometer system that achieves the necessary starlight suppression 
and actual planet detection from 5 to $20 \mu \mathrm{m}$ with optical fluxes similar to those expected from astronomical sources (typically $\sim 0.2$ photons $/ \mathrm{s} / \mathrm{m}^{2}$ for an Earth-like planet located at $10 \mathrm{pc}$ ). To achieve this goal, preliminary system studies are required to (i) define the cryogenic design for passive cooling of the optics and active cooling of the detectors; (ii) characterize and minimize the vibrations of the interferometer in cryogenic conditions; (iii) validate the cryogenic deformable mirrors, and (iv) develop spatial filters (if needed) and beam combiners that can provide the necessary performance from 6 to $20 \mu \mathrm{m}$ under cryogenic conditions. Specific developments in terms of fringe tracking (taking into account residual vibrations) and data reduction will undoubtedly be needed to reach the required level of performance in terms of starlight suppression. Dedicated developments will also be required in the field of midinfrared detectors, although the JWST legacy will be particularly useful in this context.

\section{Conclusions}

Mid-infrared space-based interferometry is a technology of direct imaging that can uniquely characterise the atmospheres of terrestrial exoplanets around nearby main-sequence stars. The use of formation-flying telescopes makes it possible to observe and study a wide variety of planetary systems, including HZ terrestrial planets around $\mathrm{M}$ dwarfs such as Proxima b (Defrère et al 2018). Currently, no other technology can obtain mid-infrared spectra of a statistically-meaningful number of temperate rocky exoplanets, which is required to make progress towards the search for life in the Universe. Significant investments would have to be made today in order to ensure that the development of such an instrument is possible in the not too distant future.

\section{Acknowledgements}

This work was partly funded by the European Research Council under the European Union's Seventh Framework Program (ERC Grant Agreement n. 337569) and by the French Community of Belgium through an ARC grant for Concerted Research Action. DD and OA acknowledge funding from the FRSFNRS. Some of research described in this publication was carried out in part at the Jet Propulsion Laboratory, California Institute of Technology, under a contract with the National Aeronautics and Space Administration. Part of this work has been carried out within the frame of the National Center for Competence in Research PlanetS supported by the Swiss National Science Foundation. SPQ acknowledges the financial support of the SNSF. NCS was supported by Fundação para a Ciência e a Tecnologia (FCT, Portugal) through national funds and by FEDER through COMPETE2020 in the context of the projects and grants reference UID/FIS/04434/2013 \& POCI-01-0145-FEDER007672, PTDC/FIS-AST/1526/2014 \& POCI-01-0145-FEDER-016886, and 
IF/00169/2012/CP0150/CT0002. SL acknowledges support from ERC Starting Grant n. 639248. SK acknowledges support from an STFC Rutherford Fellowship (ST/J004030/1) and ERC Starting Grant n. 639889.

\section{References}

Airapetian VS, Jackman CH, Mlynczak M, Danchi W, Hunt L (2017) Atmospheric beacons of life from exoplanets around $\mathrm{g}$ and $\mathrm{k}$ stars. Scientific Reports 7(1):14,141, DOI 10.1038/s41598-017-14192-4, URL https: //doi.org/10.1038/s41598-017-14192-4

Angel JR, Burge JH, Woolf NJ (1997) Detection and spectroscopy of exoplanets like Earth. In: Ardeberg AL (ed) Optical Telescopes of Today and Tomorrow, Proceedings SPIE, vol 2871, pp 516-519, DOI 10.1117/12.269076

Angel JRP, Woolf NJ (1997) An Imaging Nulling Interferometer to Study Extrasolar Planets. ApJ475:373-379

Angel JRP, Cheng AYS, Woolf NJ (1986) A space telescope for infrared spectroscopy of earth-like planets. Nature322:341-343, DOI 10.1038/322341a0

Beichman C, Benneke B, Knutson H, Smith R, Lagage PO, Dressing C, Latham D, Lunine J, Birkmann S, Ferruit P, Giardino G, Kempton E, Carey S, Krick J, Deroo PD, Mandell A, Ressler ME, Shporer A, Swain M, Vasisht G, Ricker G, Bouwman J, Crossfield I, Greene T, Howell S, Christiansen J, Ciardi D, Clampin M, Greenhouse M, Sozzetti A, Goudfrooij P, Hines D, Keyes T, Lee J, McCullough P, Robberto M, Stansberry J, Valenti J, Rieke M, Rieke G, Fortney J, Bean J, Kreidberg L, Ehrenreich D, Deming D, Albert L, Doyon R, Sing D (2014) Observations of Transiting Exoplanets with the James Webb Space Telescope (JWST). PASP126:1134, DOI 10.1086/679566

Boccaletti A, Lagage PO, Baudoz P, Beichman C, Bouchet P, Cavarroc C, Dubreuil D, Glasse A, Glauser AM, Hines DC, Lajoie CP, Lebreton J, Perrin MD, Pueyo L, Reess JM, Rieke GH, Ronayette S, Rouan D, Soummer R, Wright GS (2015) The Mid-Infrared Instrument for the James Webb Space Telescope, V: Predicted Performance of the MIRI Coronagraphs. PASP127:633-645, DOI 10.1086/682256, 1508.02352

Bolmont E, Libert AS, Leconte J, Selsis F (2016) Habitability of planets on eccentric orbits: Limits of the mean flux approximation. A\&A591:A106, DOI 10.1051/0004-6361/201628073, 1604.06091

Bracewell RN (1978) Detecting nonsolar planets by spinning infrared interferometer. Nature274:780, DOI 10.1038/274780a0

Brandl BR, Feldt M, Glasse A, Guedel M, Heikamp S, Kenworthy M, Lenzen R, Meyer MR, Molster F, Paalvast S, Pantin EJ, Quanz SP, Schmalzl E, Stuik R, Venema L, Waelkens C (2014) METIS: the mid-infrared E-ELT imager and spectrograph. In: Ground-based and Airborne Instrumentation for Astronomy V, Proc. SPIE, vol 9147, p 914721, DOI 10.1117/12.2056468, 1409.3087 
Brandl BR, Agocs T, Aitink-Kroes G, Bertram T, Bettonvil F, van Boekel R, Boulade O, Feldt M, Glasse A, Glauser A, Godel M, Hurtado N, Jager R, Kenworthy MA, Mach M, Meisner J, Meyer M, Pantin E, Quanz S, Schmid HM, Stuik R, Veninga A, Waelkens C (2016) DOI 10.1117/12.2233974, URL http://dx.doi.org/10.1117/12.2233974

Catling DC, Krissansen-Totton J, Kiang NY, Crisp D, Robinson TD, DasSarma S, Rushby AJ, Del Genio A, Bains W, Domagal-Goldman S (2018) Exoplanet Biosignatures: A Framework for Their Assessment. Astrobiology 18:709-738, DOI 10.1089/ast.2017.1737, 1705.06381

Cockell CS, Léger A, Fridlund M, Herbst TM, Kaltenegger L, Absil O, Beichman C, Benz W, Blanc M, Brack A, Chelli A, Colangeli L, Cottin H, Coudé du Foresto V, Danchi WC, Defrère D, den Herder JW, Eiroa C, Greaves J, Henning T, Johnston KJ, Jones H, Labadie L, Lammer H, Launhardt R, Lawson P, Lay OP, LeDuigou JM, Liseau R, Malbet F, Martin SR, Mawet D, Mourard D, Moutou C, Mugnier LM, Ollivier M, Paresce F, Quirrenbach A, Rabbia YD, Raven JA, Rottgering HJA, Rouan D, Santos NC, Selsis F, Serabyn E, Shibai H, Tamura M, Thiébaut E, Westall F, White GJ (2009) Darwin-A Mission to Detect and Search for Life on Extrasolar Planets. Astrobiology 9:1-22, DOI 10.1089/ast.2007.0227, 0805.1873

Colavita MM, Serabyn E, Millan-Gabet R, Koresko CD, Akeson RL, Booth AJ, Mennesson BP, Ragland SD, Appleby EC, Berkey BC, Cooper A, Crawford SL, Creech-Eakman MJ, Dahl W, Felizardo C, Garcia-Gathright JI, Gathright JT, Herstein JS, Hovland EE, Hrynevych MA, Ligon ER, Medeiros DW, Moore JD, Morrison D, Paine CG, Palmer DL, Panteleeva T, Smith B, Swain MR, Smythe RF, Summers KR, Tsubota K, Tyau C, Vasisht G, Wetherell E, Wizinowich PL, Woillez JM (2009) Keck Interferometer Nuller Data Reduction and On-Sky Performance. PASP121:1120-1138, DOI 10.1086/606063

Cowan NB, Voigt A, Abbot DS (2012) Thermal Phases of Earth-like Planets: Estimating Thermal Inertia from Eccentricity, Obliquity, and Diurnal Forcing. ApJ757:80, DOI 10.1088/0004-637X/757/1/80, 1205.5034

Crossfield IJM, Hansen BMS, Harrington J, Cho JYK, Deming D, Menou $\mathrm{K}$, Seager S (2010) A New $24 \mu \mathrm{m}$ Phase Curve for $v$ Andromedae b. ApJ723:1436-1446, DOI 10.1088/0004-637X/723/2/1436, 1008.0393

D'Amico S, Ardaens JS, Larsson R (2012) Spaceborne Autonomous Formation-Flying Experiment on the PRISMA Mission. Journal of Guidance Control Dynamics 35:834-850, DOI 10.2514/1.55638

Danchi W, Bailey V, Bryden G, Defrere D, Haniff C, Hinz P, Kennedy G, Mennesson G, Millan-Gabet R, Rieke G, Roberge A, Serabyn E, Skemer A, Stapelfeldt K, Weinberger A, Wyatt M (2014) The LBTI hunt for observable signatures of terrestrial systems (HOSTS) survey: a key NASA science program on the road to exoplanet imaging missions. In: Optical and Infrared Interferometry IV, Proc. SPIE, vol 9146, p 914607, DOI 10.1117/12.2056681

Danchi WC, Barry RK, Lawson PR, Traub WA, Unwin S (2008) The FourierKelvin Stellar Interferometer (FKSI): a review, progress report, and update. 
In: Optical and Infrared Interferometry, Proc. SPIE, vol 7013, p 70132Q, DOI 10.1117/12.790649

Defrère D, Absil O, den Hartog R, Hanot C, Stark C (2010) Nulling interferometry: impact of exozodiacal clouds on the performance of future lifefinding space missions. A\&A509:A9, DOI 10.1051/0004-6361/200912973, 0910.3486

Defrère D, Hinz PM, Skemer AJ, Kennedy GM, Bailey VP, Hoffmann WF, Mennesson B, Millan-Gabet R, Danchi WC, Absil O, Arbo P, Beichman C, Brusa G, Bryden G, Downey EC, Durney O, Esposito S, Gaspar A, Grenz P, Haniff C, Hill JM, Lebreton J, Leisenring JM, Males JR, Marion L, McMahon TJ, Montoya M, Morzinski KM, Pinna E, Puglisi A, Rieke G, Roberge A, Serabyn E, Sosa R, Stapeldfeldt K, Su K, Vaitheeswaran V, Vaz A, Weinberger AJ, Wyatt MC (2015) First-light LBT Nulling Interferometric Observations: Warm Exozodiacal Dust Resolved within a Few AU of $\eta$ Crv. ApJ799:42, DOI 10.1088/0004-637X/799/1/42, 1501.04144

Defrère D, Hinz PM, Mennesson B, Hoffmann WF, Millan-Gabet R, Skemer AJ, Bailey V, Danchi WC, Downey EC, Durney O, Grenz P, Hill JM, McMahon TJ, Montoya M, Spalding E, Vaz A, Absil O, Arbo P, Bailey H, Brusa G, Bryden G, Esposito S, Gaspar A, Haniff CA, Kennedy GM, Leisenring JM, Marion L, Nowak M, Pinna E, Powell K, Puglisi A, Rieke G, Roberge A, Serabyn E, Sosa R, Stapeldfeldt K, Su K, Weinberger AJ, Wyatt MC (2016) Nulling Data Reduction and On-sky Performance of the Large Binocular Telescope Interferometer. ApJ824:66, DOI 10.3847/0004-637X/824/2/66, 1601.06866

Defrère D, Absil O, Berger JP, Boulet T, Danchi WC, Ertel S, Gallenne A, Hénault F, Hinz P, Huby E, Ireland M, Kraus S, Labadie L, Le Bouquin JB, Martin G, Matter A, Mérand A, Mennesson B, Minardi S, Monnier JD, Norris B, de Xivry GO, Pedretti E, Pott JU, Reggiani M, Serabyn E, Surdej J, Tristram KRW, Woillez J (2018) The path towards high-contrast imaging with the vlti: the hi-5 project. Experimental Astronomy DOI 10.1007/ s10686-018-9593-2, URL https://doi.org/10.1007/s10686-018-9593-2

Defrère D, Léger A, Absil O, Garcia Munoz A, Grenfell JL, Godolt M, Loicq J, Kammerer J, Quanz S, Rauer H, Schifano L, Tian F (2018) Characterizing the atmosphere of Proxima b with a space-based mid-infrared nulling interferometer. ArXiv e-prints 1807.09996

Des Marais DJ, Harwit MO, Jucks KW, Kasting JF, Lin DNC, Lunine JI, Schneider J, Seager S, Traub WA, Woolf NJ (2002) Remote Sensing of Planetary Properties and Biosignatures on Extrasolar Terrestrial Planets. Astrobiology 2:153-181, DOI 10.1089/15311070260192246

Eisenhauer F, Perrin G, Brandner W, Straubmeier C, Perraut K, Amorim A, Schöller M, Gillessen S, Kervella P, Benisty M, Araujo-Hauck C, Jocou L, Lima J, Jakob G, Haug M, Clénet Y, Henning T, Eckart A, Berger JP, Garcia P, Abuter R, Kellner S, Paumard T, Hippler S, Fischer S, Moulin T, Villate J, Avila G, Gräter A, Lacour S, Huber A, Wiest M, Nolot A, Carvas P, Dorn R, Pfuhl O, Gendron E, Kendrew S, Yazici S, Anton S, Jung Y, Thiel M, Choquet É, Klein R, Teixeira P, Gitton P, Moch D, Vincent F, 
Kudryavtseva N, Ströbele S, Sturm S, Fédou P, Lenzen R, Jolley P, Kister C, Lapeyrère V, Naranjo V, Lucuix C, Hofmann R, Chapron F, Neumann U, Mehrgan L, Hans O, Rousset G, Ramos J, Suarez M, Lederer R, Reess JM, Rohloff RR, Haguenauer P, Bartko H, Sevin A, Wagner K, Lizon JL, Rabien S, Collin C, Finger G, Davies R, Rouan D, Wittkowski M, DoddsEden K, Ziegler D, Cassaing F, Bonnet H, Casali M, Genzel R, Lena P (2011) GRAVITY: Observing the Universe in Motion. The Messenger 143:16-24

Errmann R, Minardi S, Labadie L, Muthusubramanian B, Dreisow F, Nolte S, Pertsch T (2015) Interferometric nulling of four channels with integrated optics. Appl Opt54:7449, DOI 10.1364/AO.54.007449

Ertel S, Defrère D, Hinz P, Mennesson B, Kennedy GM, Danchi WC, Gelino C, Hill JM, Hoffmann WF, Rieke G, Shannon A, Spalding E, Stone JM, Vaz A, Weinberger AJ, Willems P, Absil O, Arbo P, Bailey VP, Beichman C, Bryden G, Downey EC, Durney O, Esposito S, Gaspar A, Grenz P, Haniff CA, Leisenring JM, Marion L, McMahon TJ, Millan-Gabet R, Montoya M, Morzinski KM, Pinna E, Power J, Puglisi A, Roberge A, Serabyn E, Skemer AJ, Stapelfeldt K, Su KYL, Vaitheeswaran V, Wyatt MC (2018a) The HOSTS Survey_Exozodiacal Dust Measurements for 30 Stars. AJ155:194, DOI 10.3847/1538-3881/aab717, 1803.11265

Ertel S, Kennedy GM, Defrère D, Hinz P, Shannon AB, Mennesson B, Danchi WC, Gelino C, Hill JM, Hoffmann WF, Rieke G, Spalding E, Stone JM, Vaz A, Weinberger AJ, Willems P, Absil O, Arbo P, Bailey VP, Beichman C, Bryden G, Downey EC, Durney O, Esposito S, Gaspar A, Grenz P, Haniff CA, Leisenring JM, Marion L, McMahon TJ, Millan-Gabet R, Montoya M, Morzinski KM, Pinna E, Power J, Puglisi A, Roberge A, Serabyn E, Skemer AJ, Stapelfeldt K, Su KYL, Vaitheeswaran V, Wyatt MC (2018b) The HOSTS Survey for Exozodiacal Dust: Preliminary results and future prospects. ArXiv e-prints 1807.08209

Fridlund M, Eiroa C, Henning T, Herbst T, Lammer H, Léger A, Liseau R, Paresce F, Penny A, Quirrenbach A, Röttgering H, Selsis F, White GJ, Absil O, Defrère D, Schneider J, Tinetti G, Karlsson A, Gondoin P, den Hartog R, D'Arcio L, Stankov AM, Kilter M, Erd C, Beichman C, Coulter D, Danchi W, Devirian M, Johnston KJ, Lawson P, Lay OP, Lunine J, Kaltenegger L (2010) The Search for Worlds Like Our Own. Astrobiology 10:5-17, DOI 10.1089/ast.2009.0380

Fujii Y, Angerhausen D, Deitrick R, Domagal-Goldman S, Grenfell JL, Hori Y, Kane SR, Pallé E, Rauer H, Siegler N, Stapelfeldt K, Stevenson KB (2018) Exoplanet Biosignatures: Observational Prospects. Astrobiology 18:739 778, DOI 10.1089/ast.2017.1733, 1705.07098

Hanot C, Mennesson B, Martin S, Liewer K, Loya F, Mawet D, Riaud P, Absil O, Serabyn E (2011) Improving Interferometric Null Depth Measurements using Statistical Distributions: Theory and First Results with the Palomar Fiber Nuller. ApJ729:110, DOI 10.1088/0004-637X/729/2/110, 1103.4719

Hinz PM, Defrère D, Skemer A, Bailey V, Stone J, Spalding E, Vaz A, Pinna E, Puglisi A, Esposito S, Montoya M, Downey E, Leisenring J, Durney O, Hoffmann W, Hill J, Millan-Gabet R, Mennesson B, Danchi W, Morzinski 
K, Grenz P, Skrutskie M, Ertel S (2016) Overview of LBTI: a multipurpose facility for high spatial resolution observations. In: Optical and Infrared Interferometry and Imaging V, Proc SPIE, vol 9907, p 990704, DOI 10. $1117 / 12.2233795$

Jovanovic N, Martinache F, Guyon O, Clergeon C, Singh G, Kudo T, Garrel V, Newman K, Doughty D, Lozi J, Males J, Minowa Y, Hayano Y, Takato N, Morino J, Kuhn J, Serabyn E, Norris B, Tuthill P, Schworer G, Stewart P, Close L, Huby E, Perrin G, Lacour S, Gauchet L, Vievard S, Murakami N, Oshiyama F, Baba N, Matsuo T, Nishikawa J, Tamura M, Lai O, Marchis F, Duchene G, Kotani T, Woillez J (2015) The Subaru Coronagraphic Extreme Adaptive Optics System: Enabling High-Contrast Imaging on Solar-System Scales. PASP127:890, DOI 10.1086/682989, 1507.00017

Kaltenegger L, Eiroa C, Fridlund CVM (2010) Target star catalogue for Darwin Nearby Stellar sample for a search for terrestrial planets. Ap\&SS326:233-247, DOI 10.1007/s10509-009-0223-3, 0810.5138

Kammerer J, Quanz SP (2017) Simulating the Exoplanet Yield of a Spacebased MIR Interferometer Based on Kepler Statistics. ArXiv e-prints 1707. 06820

Karlsson AL, Wallner O, Perdigues Armengol JM, Absil O (2004) Three telescope nuller based on multibeam injection into single-mode waveguide. In: Traub WA (ed) Proc. SPIE, vol 5491, pp 831-+

Kiang NY, Domagal-Goldman S, Parenteau MN, Catling DC, Fujii Y, Meadows VS, Schwieterman EW, Walker SI (2018) Exoplanet Biosignatures: At the Dawn of a New Era of Planetary Observations. Astrobiology 18:619-629, DOI 10.1089/ast.2018.1862

Knutson HA, Charbonneau D, Allen LE, Fortney JJ, Agol E, Cowan NB, Showman AP, Cooper CS, Megeath ST (2007) A map of the day-night contrast of the extrasolar planet HD 189733b. Nature447:183-186, DOI 10.1038/nature05782, 0705.0993

Ksendzov A, Lay O, Martin S, Sanghera JS, Busse LE, Kim WH, Pureza PC, Nguyen VQ, Aggarwal ID (2007) Characterization of mid-infrared single mode fibers as modal filters. Appl Opt46:7957-7962, DOI 10.1364/AO.46. 007957

Ksendzov A, Lewi T, Lay OP, Martin SR, Gappinger RO, Lawson PR, Peters RD, Shalem S, Tsun A, Katzir A (2008) Modal filtering for midinfrared nulling interferometry using single mode silver halide fibers. Appl Opt47:5728, DOI 10.1364/AO.47.005728

Lawson P, Traub W (2006) Earth-Like Exoplanets: The Science of NASA's Navigator Program. JPL Publication

Lawson PR, Lay OP, Johnston KJ, Beichman CA (2007) Terrestrial Planet Finder Interferometer Science Working Group Report. NASA STI/Recon Technical Report N 8

Lay OP (2004) Systematic Errors in Nulling Interferometers. Appl Opt43:6100-6123, DOI 10.1364/AO.43.006100

Le Bouquin JB, Berger JP, Lazareff B, Zins G, Haguenauer P, Jocou L, Kern P, Millan-Gabet R, Traub W, Absil O, Augereau JC, Benisty M, Blind 
N, Bonfils X, Bourget P, Delboulbe A, Feautrier P, Germain M, Gitton P, Gillier D, Kiekebusch M, Kluska J, Knudstrup J, Labeye P, Lizon JL, Monin JL, Magnard Y, Malbet F, Maurel D, Ménard F, Micallef M, Michaud L, Montagnier G, Morel S, Moulin T, Perraut K, Popovic D, Rabou P, Rochat S, Rojas C, Roussel F, Roux A, Stadler E, Stefl S, Tatulli E, Ventura N (2011) PIONIER: a 4-telescope visitor instrument at VLTI. A\&A535:A67, DOI 10.1051/0004-6361/201117586, 1109.1918

Léger A, Mariotti JM, Mennesson B, Ollivier M, Puget JL, Rouan D, Schneider J (1996) The DARWIN project. Ap\&SS241:135-146, DOI 10.1007/ BF00644221

Léger A, Fontecave M, Labeyrie A, Samuel B, Demangeon O, Valencia D (2011) Is the Presence of Oxygen on an Exoplanet a Reliable Biosignature? Astrobiology 11:335-341, DOI 10.1089/ast.2010.0516

Martin S, Booth A, Liewer K, Raouf N, Loya F, Tang H (2012) High performance testbed for four-beam infrared interferometric nulling and exoplanet detection. Appl Opt51:3907-3921, DOI 10.1364/AO.51.003907

Martin S, Serabyn G, Liewer K, Mennesson B (2017) Achromatic broadband nulling using a phase grating. Optica 4(1):110-113, DOI 10.1364/OPTICA. 4.000110, URL http://www.osapublishing.org/optica/abstract.cfm? URI=optica-4-1-110

Martin SR, Booth AJ (2010) Demonstration of exoplanet detection using an infrared telescope array. A\&A520:A96, DOI 10.1051/0004-6361/201014942

Maurin AS, Selsis F, Hersant F, Belu A (2012) Thermal phase curves of nontransiting terrestrial exoplanets. II. Characterizing airless planets. A\&A538:A95, DOI 10.1051/0004-6361/201117054, 1110.3087

Meadows VS, Reinhard CT, Arney GN, Parenteau MN, Schwieterman EW, Domagal-Goldman SD, Lincowski AP, Stapelfeldt KR, Rauer H, DasSarma S, Hegde S, Narita N, Deitrick R, Lustig-Yaeger J, Lyons TW, Siegler N, Grenfell JL (2018) Exoplanet Biosignatures: Understanding Oxygen as a Biosignature in the Context of Its Environment. Astrobiology 18:630-662, DOI 10.1089/ast.2017.1727, 1705.07560

Mennesson B, Mariotti JM (1997) Array Configurations for a Space Infrared Nulling Interferometer Dedicated to the Search for Earthlike Extrasolar Planets. Icarus128:202-212, DOI 10.1006/icar.1997.5731

Mennesson B, Ollivier M, Ruilier C (2002) Use of single-mode waveguides to correct the optical defects of a nulling interferometer. Journal of the Optical Society of America A 19:596-602, DOI 10.1364/JOSAA.19.000596

Mennesson B, Hanot C, Serabyn E, Liewer K, Martin SR, Mawet D (2011a) High-contrast Stellar Observations within the Diffraction Limit at the Palomar Hale Telescope. ApJ743:178, DOI 10.1088/0004-637X/743/2/178

Mennesson B, Serabyn E, Hanot C, Martin SR, Liewer K, Mawet D (2011b) New Constraints on Companions and Dust within a Few AU of Vega. ApJ736:14, DOI 10.1088/0004-637X/736/1/14

Mennesson B, Millan-Gabet R, Serabyn E, Colavita MM, Absil O, Bryden G, Wyatt M, Danchi W, Defrère D, Doré O, Hinz P, Kuchner M, 
Ragland S, Scott N, Stapelfeldt K, Traub W, Woillez J (2014) Constraining the Exozodiacal Luminosity Function of Main-sequence Stars: Complete Results from the Keck Nuller Mid-infrared Surveys. ApJ797:119, DOI 10.1088/0004-637X/797/2/119

Monnier JD, Ireland MJ, Kraus S, Baron F, Creech-Eakman M, Dong R, Isella A, Merand A, Michael E, Minardi S, Mozurkewich D, Petrov R, Rinehart S, ten Brummelaar T, Vasisht G, Wishnow E, Young J, Zhu Z (2016) Architecture design study and technology road map for the Planet Formation Imager (PFI). In: Society of Photo-Optical Instrumentation Engineers (SPIE) Conference Series, Proceedings SPIE, vol 9907, p 99071O, DOI $10.1117 / 12.2233311,1608.00580$

Moskovitz NA, Gaidos E, Williams DM (2009) The Effect of Lunarlike Satellites on the Orbital Infrared Light Curves of Earth-Analog Planets. Astrobiology 9:269-277, DOI 10.1089/ast.2007.0209, 0810.2069

Ollivier M, Absil O, Allard F, Berger JP, Bordé P, Cassaing F, Chazelas B, Chelli A, Chesneau O, Coudé du Foresto V, Defrère D, Duchon P, Gabor P, Gay J, Herwats E, Jacquinod S, Kern P, Kervella P, Le Duigou JM, Léger A, Lopez B, Malbet F, Mourard D, Pelat D, Perrin G, Rabbia Y, Rouan D, Reiss JM, Rousset G, Selsis F, Stee P, Surdej J (2009) PEGASE, an infrared interferometer to study stellar environments and low mass companions around nearby stars. Experimental Astronomy 23:403-434, DOI 10.1007/s10686-008-9133-6

Peters RD, Lay OP, Lawson PR (2010) Mid-Infrared Adaptive Nulling for the Detection of Earthlike Exoplanets. PASP122:85-92, DOI 10.1086/649850

Quanz SP, Crossfield I, Meyer MR, Schmalzl E, Held J (2015) Direct detection of exoplanets in the 3-10 $\mu \mathrm{m}$ range with E-ELT/METIS. International Journal of Astrobiology 14:279-289, DOI 10.1017/S1473550414000135, 1404. 0831

Quanz SP, Kammerer J, Defrère D, Absil O, Glauser AM, Kitzmann D (2018) Exoplanet science with a space-based mid-infrared nulling interferometer. ArXiv e-prints 1807.06088

Rinehart SA, Savini G, Holland W, Absil O, Defrère D, Spencer L, Leisawitz D, Rizzo M, Juanola-Paramon R, Mozurkewich D (2016) The path to interferometry in space. In: Society of Photo-Optical Instrumentation Engineers (SPIE) Conference Series, Proc. SPIE, vol 9907, p 99070S, DOI $10.1117 / 12.2231754$

Schwieterman EW, Kiang NY, Parenteau MN, Harman CE, DasSarma S, Fisher TM, Arney GN, Hartnett HE, Reinhard CT, Olson SL, Meadows VS, Cockell CS, Walker SI, Grenfell JL, Hegde S, Rugheimer S, Hu R, Lyons TW (2018) Exoplanet Biosignatures: A Review of Remotely Detectable Signs of Life. Astrobiology 18:663-708, DOI 10.1089/ast.2017.1729, 1705.05791

Seager S, Bains W, Petkowski JJ (2016) Toward a List of Molecules as Potential Biosignature Gases for the Search for Life on Exoplanets and Applications to Terrestrial Biochemistry. Astrobiology 16:465-485, DOI 10.1089 /ast.2015.1404 
Selsis F (2004) The Atmosphere of Terrestrial Exoplanets: Detection and Characterization. In: Beaulieu J, Lecavelier Des Etangs A, Terquem C (eds) Extrasolar Planets: Today and Tomorrow, Astronomical Society of the Pacific Conference Series, vol 321, p 170

Selsis F, Wordsworth RD, Forget F (2011) Thermal phase curves of nontransiting terrestrial exoplanets. I. Characterizing atmospheres. A\&A532:A1, DOI 10.1051/0004-6361/201116654, 1104.4763

Selsis F, Maurin AS, Hersant F, Leconte J, Bolmont E, Raymond SN, Delbo' M (2013) The effect of rotation and tidal heating on the thermal lightcurves of super Mercuries. A\&A555:A51, DOI 10.1051/0004-6361/201321661, 1305. 3858

Stevenson KB, Désert JM, Line MR, Bean JL, Fortney JJ, Showman AP, Kataria T, Kreidberg L, McCullough PR, Henry GW, Charbonneau D, Burrows A, Seager S, Madhusudhan N, Williamson MH, Homeier D (2014) Thermal structure of an exoplanet atmosphere from phase-resolved emission spectroscopy. Science 346:838-841, DOI 10.1126/science.1256758, 1410.2241

von Paris P, Hedelt P, Selsis F, Schreier F, Trautmann T (2013) Characterization of potentially habitable planets: Retrieval of atmospheric and planetary properties from emission spectra. A\&A551:A120, DOI 10.1051/0004-6361/ 201220009, 1301.0217

Wallner O, Leeb WR, Winzer PJ (2002) Minimum length of a single-mode fiber spatial filter. Journal of the Optical Society of America A 19:24452448, DOI 10.1364/JOSAA.19.002445

Weber V, Barillot M, Haguenauer P, Kern PY, Schanen-Duport I, Labeye PR, Pujol L, Sodnik Z (2004) Nulling interferometer based on an integrated optics combiner. In: Traub WA (ed) New Frontiers in Stellar Interferometry, Proc. SPIE, vol 5491, p 842

Winn JN, Fabrycky DC (2015) The Occurrence and Architecture of Exoplanetary Systems. ARA\&A53:409-447, DOI 10.1146/annurev-astro-082214-122246, 1410.4199 